Research Article

\title{
Research on Fuzzy Plastic Constitutive Model Based on Membership Function
}

\author{
Xigang Wang $\mathbb{D},{ }^{1}$ Liling Jin $\mathbb{D}{ }^{1}$ and Mingfu Fu $\mathbb{D}^{2}$ \\ ${ }^{1}$ School of Resources and Civil Engineering, Liaoning Institute of Science and Technology, Xihu District, Benxi 117004, China \\ ${ }^{2}$ School of Civil Engineering and Architecture, Nanchang University, Honggutan District, Nanchang 330031, China \\ Correspondence should be addressed to Xigang Wang; fx_wxg@lnist.edu.cn
}

Received 22 June 2021; Revised 7 October 2021; Accepted 21 October 2021; Published 2 November 2021

Academic Editor: Lei Hou

Copyright ( 2021 Xigang Wang et al. This is an open access article distributed under the Creative Commons Attribution License, which permits unrestricted use, distribution, and reproduction in any medium, provided the original work is properly cited.

Soil has no obvious yield point, and the classical elastoplastic theory contradicts the uncertainty of the plastic yield point of the soil. Therefore, a fuzzy plastic Cambridge model based on the membership function was designed by combining the fuzzy mathematics with the Cambridge model. This model made the plastic membership function to correspond with the fuzzy yield function. The plastic strain at any stress state was calculated using the fuzzy Cambridge model and was compared with the indoor triaxial test results, and they were in good agreement. Therefore, it is appropriate to use fuzzy mathematics to express the unobvious soil yield property. The characteristics of soil yield in any stress state is reflected by the fuzzy plastic theory, which indicates that there is entirely no elasticity at any stress state. Moreover, the varying degrees of plasticity and the degree of plastic yield were uniquely determined by the plastic membership function. The fuzzy plastic model used the membership function change to replace the complex hardening. Additionally, the cyclic loading path was clear and appropriate for the cyclic loading and unloading calculations.

\section{Introduction}

The elastoplastic theory states that the plastic deformation of a material meets a certain yield condition. The elastic and plastic deformations are controlled by the yield condition, and the material's yield point is determined. Numerous experiments have revealed that there is no apparent distinction between the elasticity and plasticity of the soil. Therefore, it is inappropriate to use classic yield conditions to handle the plastic deformation of soil materials, and the effective approach is to use fuzzy mathematics to solve the uncertainty of the soil yield point.

Many engineering structures and components often operate under low cycle fatigue conditions. Cyclic load and cyclic plastic strain are the root causes of material fatigue. Therefore, the problem of cyclic strain analysis of elasticplastic materials is worth exploring.

The stress point, presented in Figure 1, produced only elastic strain. The plastic strain occurred in accordance with the hardening rule only when the stress point reached the yield line $f\left(p, q, \varepsilon_{v}^{p}\right)=0$. The technique to reflect the plastic strain at any point in the elastic region depicted in Figure 1 is a problem that needs to be solved. The stress state within the yield surface did not produce plastic strain to solve this problem Hashiguch [1] proposed a lower load surface concept. Dafalias [2-4] proposed a boundary surface model to calculate the plasticity in the initial yield surface. Klisinski [5] proposed applying fuzzy mathematics to plastic mechanics theory and established the basic framework of fuzzy plastic mechanics. Jiang [6] analyzed the fuzzy factors in elastoplastic mechanics and explained the ambiguity of the yield and failure criterion of concrete materials. Moreover, he proposed the idea of describing the yield state with a membership function. Fu et al. established and demonstrated the uniqueness and existence of the fuzzy elastoviscoplastic constitutive model along with its solution and studied the continuous transition of elastoplasticity in statics [7, 8]. Wang et al. [9-11] studied the plastic membership function of geotechnical media. They obtained a fuzzy plastic constitutive model suitable for geotechnical media and 


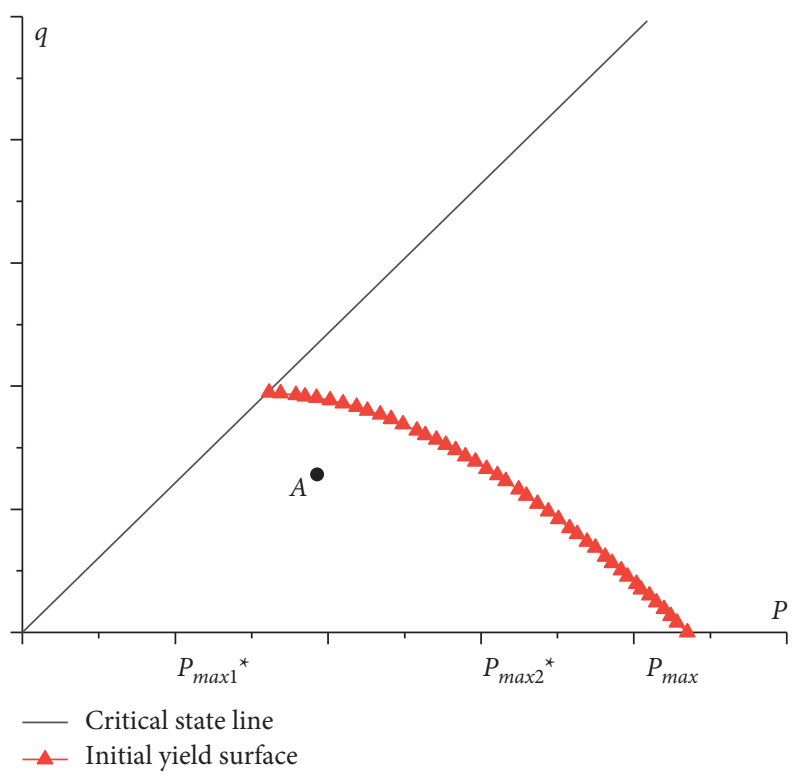

Figure 1: The elastic area of the Cambridge model.

provided a general method for transforming the plastic model into a fuzzy plastic model. Considering the complexity of geotechnical materials, many scholars [12-15] used fuzzy mathematics to analyze geotechnical problems. Landgraf used a multistage load test method to describe the cyclic response of the material. Morrow described the cyclic stress-strain relationship with analytical expressions; however, the expression was not accurate enough to reflect the strength and weakness of the material. Li [16] proposed an improved boundary surface model of remolded clay that considered the elastoplastic loading and unloading processes under cyclic loading. The model adopted a simple boundary surface form, which oversimplified the derivation and calculation of the model theory. Based on the existing constitutive model, Zhao [17] proposed a cyclic boundary surface constitutive model of saturated sand. Zhou [18] proposed a secondary loading surface model suitable for cyclic loading and unloading of rocks based on the Drucker-Prager yield criterion combined with the secondary loading surface. Huang [19] proposed an elastoplastic constitutive model using the boundary surface plasticity theory and described the mechanical properties of unsaturated soil under cyclic loading. Based on the movable hardening criterion of the hardening center and boundary surface, Yao [20] described the anisotropy of soil caused by cyclic loading. Furthermore, he proposed a detailed plastic modulus interpolation method that enabled the model to accurately describe the cyclic stability of saturated clay under low stress levels. Instead of the plastic volumetric strain increment, Dong [21] used hardening parameters independent of the stress path to modify the yield surface of the modified Cambridge model.

The soil yield is unobvious, and the calculation of the cyclic loading and unloading processes is complicated. In this study, we introduce the plastic membership function into the Cambridge model to obtain the fuzzy plastic Cambridge model. The fuzzy plastic Cambridge model is used to describe the fuzziness of the soil yield and solve the problems of cyclic loading and unloading. The fuzzy plastic model uses the change in the fuzzy cone surface to distinctly reflect the loading and unloading paths during cyclic loading and unloading. The continuous change in the plastic membership function can replace the complicated hardening law; therefore, the fuzzy plastic model is more suitable for cyclic loading and unloading problems.

\section{Fuzzy Yield Function}

2.1. Yield Function Fuzzification. The critical state of the Cambridge model is defined as

$$
q=M * p .
$$

According to the energy equation and the associated flow law, the plastic potential function of the Cambridge model has the same form as the yield function:

$$
f=M \ln p+\frac{q}{p}+C=0,
$$

where $C$ is a constant.

When $q=0$, the corresponding intersection point of the classical initial yield surface and the $p$-axis is $p_{\max }$, it can be obtained from equation (2) that $C=-M \ln p_{\max }$, and equation (2) becomes

$$
f=M \ln p+\frac{q}{p}-M \ln p_{\max }=0 .
$$

According to equation (3), we obtain

$$
\ln p_{\max }=\ln p+\frac{1}{M} \frac{q}{p} \text {. }
$$

The stress points in the elastic region, presented in Figure 1, cannot satisfy equation (4). Moreover, to make the stress points in the elastic region meet the corresponding 


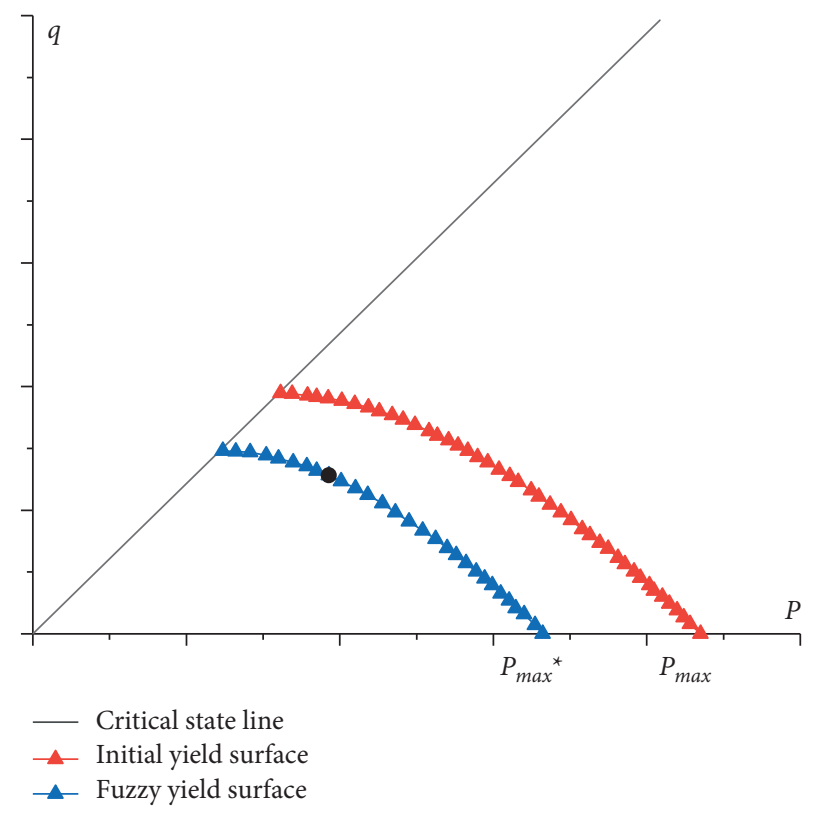

FIGURE 2: Fuzzy yield surface.

yield function, we need to shift the yield function and $p_{\max }$ to the left at a certain intersection point $p_{\max }^{*}$. Subsequently, the original stress point in the elastic region is positioned on the corresponding fuzzy yield surface, as shown in Figure 2. Therefore, the fuzzy yield function can be expressed as

$$
f^{*}=M \ln p+\frac{q}{p}-M \ln p_{\max }^{*}=0
$$

According to equation (5), when $p_{\max }^{*}$ moves from right to left, the stress point located in the elastic region (depicted in Figure 1) is positioned on a unique fuzzy yield function $f^{*}$ based on the stress state.

2.2. Plastic Membership Function. Fuzzy plasticity theory states that the stress state and test parameters determine only the fuzzy yield function established in the classical initial yield function. The stress-state parameter of the fuzzy yield function is $p_{\max }^{*}$, as shown in Figure 2. Furthermore, as shown in Figure 3, it can also be a combination of stress invariants $I_{1}$ and $J_{2}$, which represents the size of the hydrostatic pressure axis and deviation on the $\pi$ plane, respectively. It can also be a combination of other stress or strain invariants. The yield degree (plastic strain) of a fuzzy yield function is determined by the plastic membership function $\mu$ that corresponds to the fuzzy yield function and $\mu$ is determined by the amount of the stress state that represents $f^{*}$.

According to the fuzzy plasticity theory, any stress point in the elastic region depicted in Figure 1 is in a yielding state; however, the degree of yielding varies. There is no absolute elasticity or plasticity in the fuzzy plasticity theory, and it can effectively reflect the elastic region's degree of plasticity in the classical initial yield plane. The fuzzy plastic membership function adopts the assigned plastic membership function form (of course, numerical algorithms such as inversion analysis can be used to determine a more realistic form) to explain the fuzzy plasticity theory. The stress-state parameter adopts $p_{\max }^{*}$, and the plastic membership function form is

$$
\mu=\left(\frac{p_{\max }^{*}}{p_{\max }}\right)^{\alpha} .
$$

In equation (6), $\alpha$ is the model parameter, which can be determined experimentally.

Explanation of fuzzy plastic membership function:

(1) When $\mu=0$, the fuzzy yield function is the initial yield function of loading, and the yield degree is $0 \%$

(2) When $\mu=1$, the fuzzy yield function is the classical initial yield function, and the yield degree is $100 \%$

(3) When $0 \%<\mu<100 \%$, the fuzzy yield function is between the initial yield function of loading and the classical initial yield function, and the yield degree is $\mu * 100 \%$

According to equations (5) and (6), $p_{\max }^{*}$ determines a plastic membership function value and a fuzzy plastic yield function, and the calculation results of the plastic membership function are presented in Table 1.

The relationship between the different plastic membership function parameters and stress is shown in Figure 4.

As depicted in Table 1 and Figure 4, the corresponding stress and plastic membership function evolution laws are different for different $\alpha$. When $\alpha<1$, the plastic membership function and stress exhibited a curved growth relationship; however, the slope kept decreasing. When $\alpha=1$, the plastic membership function had a linear growth relationship with stress. Furthermore, when $\alpha>1$, the plastic membership function and stress had a curved growth relationship, and the slope continued to increase. Additionally, with the increase in $p_{\max }^{*}$, the plastic membership function continuously increased from $0 \%$ to $100 \%$, and the degree of plasticity 


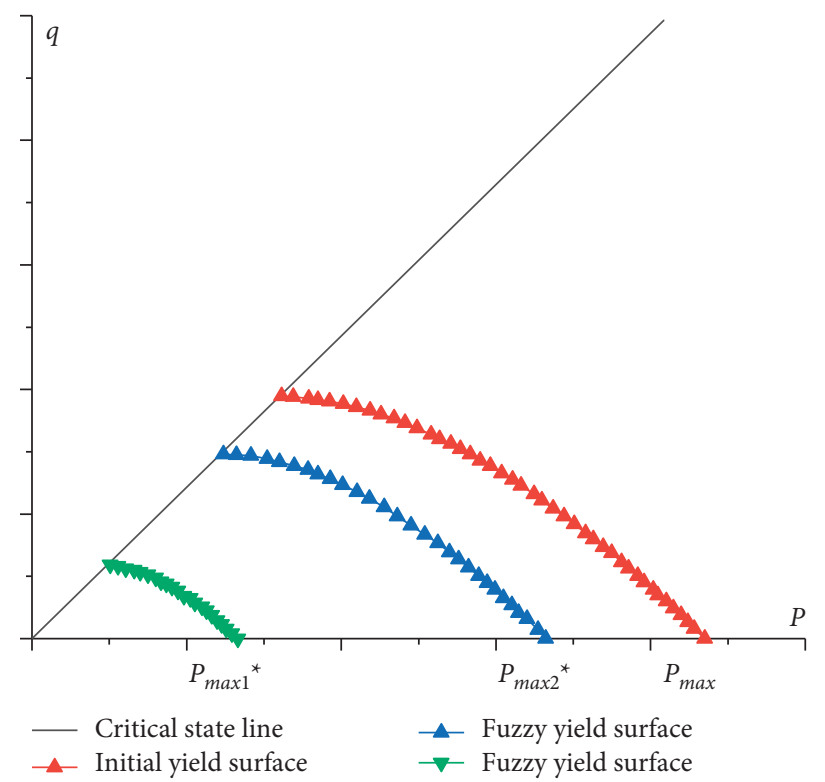

Figure 3: Evolution of fuzzy yield surface.

TABLE 1: Plastic membership function table.

\begin{tabular}{|c|c|c|c|c|c|c|c|}
\hline \multirow{2}{*}{$p_{\max }^{*}$} & \multirow{2}{*}{$p_{\max }$} & \multirow{2}{*}{$p_{\max }^{*} / p_{\max }$} & \multicolumn{5}{|c|}{$\mu(\%)$} \\
\hline & & & $\alpha=0.5$ & $\alpha=1$ & $\alpha=2$ & $\alpha=5$ & $\alpha=10$ \\
\hline 1.6 & 188 & 0.0081 & 9.21 & 0.85 & 0.01 & 0.00 & 0.00 \\
\hline 10 & 188 & 0.0510 & 23.06 & 5.32 & 0.28 & 0.00 & 0.00 \\
\hline 20 & 188 & 0.1020 & 32.62 & 10.64 & 1.13 & 0.00 & 0.00 \\
\hline 30 & 188 & 0.1531 & 39.95 & 15.96 & 2.55 & 0.01 & 0.00 \\
\hline 40 & 188 & 0.2041 & 46.13 & 21.28 & 4.53 & 0.04 & 0.00 \\
\hline 50 & 188 & 0.2551 & 51.57 & 26.60 & 7.07 & 0.13 & 0.00 \\
\hline 60 & 188 & 0.3061 & 56.49 & 31.91 & 10.19 & 0.33 & 0.00 \\
\hline 70 & 188 & 0.3571 & 61.02 & 37.23 & 13.86 & 0.72 & 0.01 \\
\hline 80 & 188 & 0.4082 & 65.23 & 42.55 & 18.11 & 1.40 & 0.02 \\
\hline 90 & 188 & 0.4592 & 69.19 & 47.87 & 22.92 & 2.51 & 0.06 \\
\hline 100 & 188 & 0.5102 & 72.93 & 53.19 & 28.29 & 4.26 & 0.18 \\
\hline 110 & 188 & 0.5612 & 76.49 & 58.51 & 34.23 & 6.86 & 0.47 \\
\hline 120 & 188 & 0.6122 & 79.89 & 63.83 & 40.74 & 10.60 & 1.12 \\
\hline 130 & 188 & 0.6633 & 83.16 & 69.15 & 47.82 & 15.81 & 2.50 \\
\hline 140 & 188 & 0.7143 & 86.29 & 74.47 & 55.45 & 22.90 & 5.24 \\
\hline 150 & 188 & 0.7653 & 89.32 & 79.79 & 63.66 & 32.33 & 10.46 \\
\hline 160 & 188 & 0.8163 & 92.25 & 85.11 & 72.43 & 44.65 & 19.94 \\
\hline 170 & 188 & 0.8673 & 95.09 & 90.43 & 81.77 & 60.46 & 36.55 \\
\hline 180 & 188 & 0.9184 & 97.85 & 95.74 & 91.67 & 80.46 & 64.74 \\
\hline 185 & 188 & 0.9439 & 99.20 & 98.40 & 96.83 & 92.27 & 85.14 \\
\hline 188 & 188 & 1.0000 & 100.00 & 100.00 & 100.00 & 100.00 & 100.00 \\
\hline
\end{tabular}

increased from low to high. In the same stress state, the plastic strain and value of $\alpha$ were inversely proportional; the larger the plastic strain, the smaller the value of $\alpha$, which was determined experimentally.

2.3. Fuzzy Plastic Strain. The fuzzy plastic Cambridge model adopted the associated flow law, and the plastic hardening parameter was the plastic volume strain $\varepsilon_{v}^{p}$. The plastic strain increment was orthogonal to the plastic potential surface. The results based on the isotropic consolidation test are shown in Figure 5. The initial void ratio is $e_{0}$, and the slopes of the loading and unloading curves are $\lambda$ and $\kappa$, respectively.

The change in void ratio for the load from any point in the elastic region depicted in Figure 1 is

$$
\Delta e=e-e_{0}=-\lambda \ln \frac{p_{\max }^{*}}{p_{0}} .
$$

The total volumetric strain can be expressed as

$$
\varepsilon_{v}=\frac{-\Delta e}{1+e_{0}}=\frac{\lambda}{1+e_{0}} \ln \frac{p_{\max }^{*}}{p_{0}} .
$$




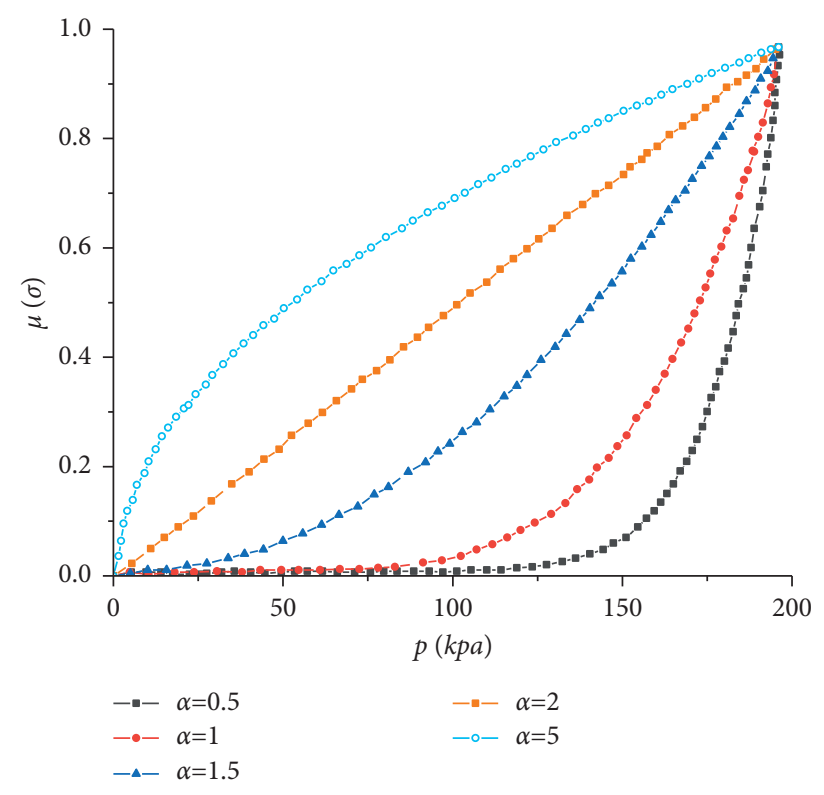

FIGURE 4: Evolution of plastic membership function.

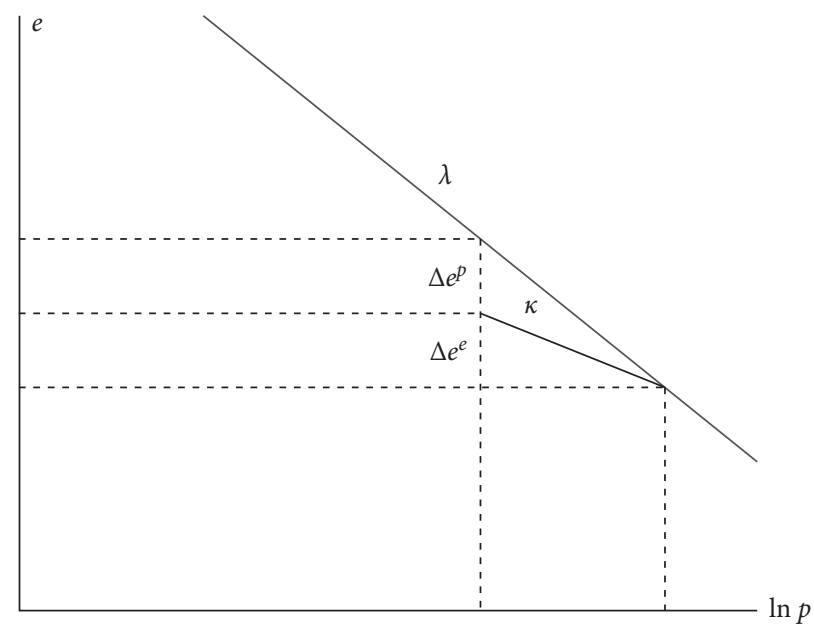

Figure 5: Changes in void ratio.

From equation (8), we can see

$$
\begin{gathered}
\varepsilon_{v}^{e}=\frac{\kappa}{1+e_{0}}\left(\ln p_{\max }^{*}-\ln p_{0}\right), \\
\varepsilon_{v}^{p}=\frac{\lambda-\kappa}{1+e_{0}}\left(\ln p_{\max }^{*}-\ln p_{0}\right),
\end{gathered}
$$

where $\varepsilon_{v}^{p}$ is the corresponding plastic volume strain of the fuzzy yield function and $f^{*}$ is the hardening parameter of the fuzzy plastic Cambridge model.

$$
\ln p_{\max }^{*}=\frac{1+e_{0}}{\lambda-\kappa} \varepsilon_{v}^{p}+\ln p_{0} .
$$

Substituting equation (11) in (5), we obtain

$$
M \ln p+\frac{q}{p}-M\left(\frac{1+e_{0}}{\lambda-\kappa} \varepsilon_{v}^{p}+\ln p_{0}\right)=0 .
$$

Sorting equation (12), we obtain

$$
\frac{\lambda-\kappa}{1+e_{0}} \ln p+\frac{1}{M} \frac{\lambda-\kappa}{1+e_{0}} \frac{1}{M} \frac{q}{p}-\varepsilon_{v}^{p}-\frac{\lambda-\kappa}{1+e_{0}} \ln p_{0}=0 .
$$

Let $c_{p}=\lambda-\kappa / 1+e_{0}$; then, the fuzzy yield function in equation (12) becomes

$$
C_{p} \ln p+\frac{1}{M} C_{p} \frac{1}{M} \frac{q}{p}-\varepsilon_{v}^{p}-C_{p} \ln p_{0}=0 .
$$

According to the consistency conditions, we can derive

$$
\frac{\partial f^{*}}{\partial p} \mathrm{~d} p+\frac{\partial f^{*}}{\partial q} \mathrm{~d} q+\frac{\partial f^{*}}{\partial \varepsilon_{v}^{p}} \mathrm{~d} \varepsilon_{v}^{p}=0 .
$$

According to equation (14), we know 


$$
\frac{\partial f^{*}}{\partial \varepsilon_{v}^{p}}=-1
$$

According to the associated flow law, the plastic potential function is orthogonal to the direction of the plastic strain increment, and the fuzzy plastic volume strain can be represented as

$$
\mathrm{d} \varepsilon_{v}^{p}=\Lambda \frac{\partial g}{\partial p}
$$

where $\Lambda$ is the plastic scalar factor, which represents the size of the plastic strain increment, and $\partial g / \partial p$ is the development direction of the plastic strain increment. Substituting equations (16) and (17) in (15), we obtain

$$
\frac{\partial f^{*}}{\partial p} \mathrm{~d} p+\frac{\partial f^{*}}{\partial q} \mathrm{~d} q-\Lambda^{*} \frac{\partial g}{\partial p}=0
$$

According to equation (18), the plastic scalar factor is

$$
\Lambda=\frac{\left(\partial f^{*} / \partial p\right) \mathrm{d} p+\left(\partial f^{*} / \partial p\right) \mathrm{d} q}{\partial g / \partial p}
$$

According to the relationship between the plastic potential function and the fuzzy yield function of the Cambridge model, from equation (14), we obtain

$$
\begin{aligned}
\frac{\partial f^{*}}{\partial p} & =C_{p} \frac{1}{p}-C_{p} \frac{1}{M} \frac{q}{p^{2}}, \\
\frac{\partial f^{*}}{\partial q} & =C_{p} \frac{1}{M} \frac{1}{p}, \\
\frac{\partial g}{\partial p} & =\frac{\partial f^{*}}{\partial p}=C_{p} \frac{1}{p}-C_{p} \frac{1}{M} \frac{q}{p^{2}} .
\end{aligned}
$$

Substituting equations (20)-(22) in (19), $\Lambda$ can be obtained as

$$
\Lambda=\frac{\left[C_{p}(1 / p)-C_{p}(1 / M)\left(q / p^{2}\right)\right] \mathrm{d} p+\left[C_{p}(1 / M)(1 / p)\right] \mathrm{d} q}{C_{p}(1 / p)-C_{p}(1 / M)\left(q / p^{2}\right)} .
$$

Substituting equations (22) and (23) in (17), the plastic strain increment $\mathrm{d} \varepsilon_{v}^{p}$ can be obtained as

$$
\mathrm{d} \varepsilon_{v}^{p}=\left[C_{p}(1 / p)-C_{p}(1 / M)\left(q / p^{2}\right)\right] \mathrm{d} p+\left[C_{p}(1 / M)(1 / p)\right] \mathrm{d} q .
$$

According to the Cambridge model equation (22), the relationship between the stress ratio and strain increment is

$$
\frac{q}{p}=M-\frac{d \varepsilon_{v}^{p}}{d \varepsilon_{d}^{p}}
$$

where $\mathrm{d} \varepsilon_{d}^{p}$ is the plastic shear strain increment.

Substituting equation (24) in (25), $\mathrm{d} \varepsilon_{d}^{p}$ can be obtained as follows:

$$
\begin{aligned}
d \varepsilon_{d}^{p}= & \frac{1}{M-(q / p)}\left[\left[C_{p}(1 / p)-C_{p}(1 / M)\left(q / p^{2}\right)\right] \mathrm{d} p\right. \\
& \left.+\left[C_{p}(1 / M)(1 / p)\right] \mathrm{d} q\right] .
\end{aligned}
$$

According to equations (24) and (26), the fuzzy plastic volumetric strain increment $d\left(\varepsilon_{v}^{p}\right)^{*}$ and the fuzzy plastic shear strain increment $d\left(\varepsilon_{d}^{p}\right)^{*}$ in the elastic region can be obtained as

$$
\left\{\begin{array}{l}
d\left(\varepsilon_{v}^{p}\right)^{*}=\mu d \varepsilon_{v}^{p}=\mu\left[C_{p}(1 / p)-C_{p}(1 / M)\left(q / p^{2}\right)\right] \mathrm{d} p+\left[C_{p}(1 / M)(1 / p)\right] \mathrm{d} q, \\
d\left(\varepsilon_{d}^{p}\right)^{*}=\mu d \varepsilon_{d}^{p}=\mu \frac{1}{M-(q / p)}\left[\left[C_{p}(1 / p)-C_{p}(1 / M)\left(q / p^{2}\right)\right] \mathrm{d} p+\left[C_{p}(1 / M)(1 / p)\right] \mathrm{d} q\right] .
\end{array}\right.
$$

The elastic strain increment was calculated in accordance with elastic theory. After deriving the Cambridge model, the elastic strain increment can be expressed as

$$
\left\{\begin{array}{l}
\mathrm{d} \varepsilon_{v}^{e}=\frac{\kappa}{1+e_{0}} \frac{1}{p} \mathrm{~d} p \\
\mathrm{~d} \varepsilon_{d}^{e}=\frac{2}{9} \frac{\kappa}{1+e_{0}} \frac{1+v}{1-2 v} \frac{1}{p} \mathrm{~d} q
\end{array}\right.
$$

The total strain increment was composed of the elastic strain increment $\mathrm{d} \varepsilon^{e}$ and the fuzzy plastic strain increment $\mathrm{d}\left(\varepsilon^{p}\right)^{*}$, which can be expressed as

$$
\left\{\begin{array}{l}
\mathrm{d} \varepsilon_{v}=\mathrm{d} \varepsilon_{v}^{e}+d\left(\varepsilon_{v}^{p}\right)^{*} \\
\mathrm{~d} \varepsilon_{d}=\mathrm{d} \varepsilon_{d}^{e}+d\left(\varepsilon_{d}^{p}\right)^{*}
\end{array}\right.
$$

\section{Analysis of Calculation Results}

3.1. Model Verification and Parameter Determination. The soil properties of a certain cohesive soil are listed in Table 2. A static triaxial test on the cohesive soil was conducted using a geotechnical triaxial test system, as shown in Figure 6 . The cylindrical soil sample had a diameter of $39.1 \mathrm{~mm}$ and a height of $80 \mathrm{~mm}$. To ensure the uniformity of the sample, it was compacted in five layers using the wet ramming method. In the triaxial pressure chamber, the soil 
TABLE 2: Cohesive soil parameters.

\begin{tabular}{ccccccccc}
\hline$e_{0}$ & $\rho\left(\mathrm{g} / \mathrm{cm}^{3}\right)$ & $\nu$ & $E(\mathrm{Gpa})$ & $C(\mathrm{Kpa})$ & $\phi\left({ }^{0}\right)$ & $\lambda$ & $\kappa$ & $M$ \\
\hline 0.65 & 1.936 & 0.252 & 4.565 & 35 & 34 & 0.0865 & 0.0185 & 1.33 \\
\hline
\end{tabular}

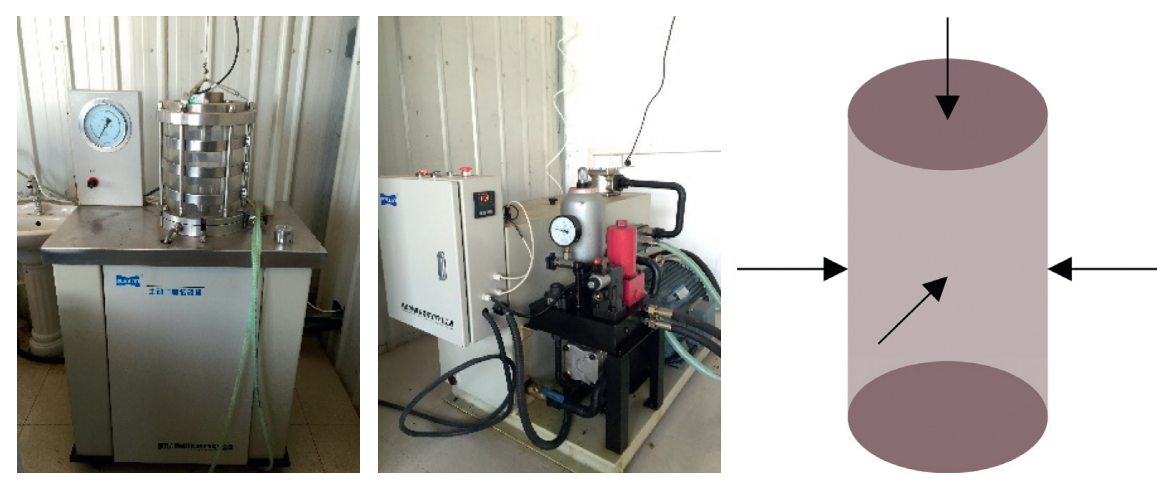

FIgURE 6: Triaxial test equipment.

sample was saturated by applying back pressure and airless water. The specimens were consolidated under different confining pressures for $24 \mathrm{~h}$ in each direction. After consolidation was completed, a static triaxial test was performed under the confining pressures of $30 \mathrm{kPa}, 60 \mathrm{kPa}$, and $90 \mathrm{kPa}$ to verify the reliability of the model.

A comparison between the triaxial test results and the calculation results of the fuzzy plastic Cambridge model is shown in Figure 7.

Figure 7 reveals that the triaxial test results are consistent with the calculation results of the fuzzy plastic Cambridge model. The test result is between $\alpha=1$ and $\alpha=1.2$ of the plastic membership function, and when $\alpha=1.2$, the test result is closer to the calculation result of the fuzzy plastic Cambridge model. The model parameters can be determined by comparing the experiments and models.

3.2. Calculation of the Model. According to equation (22), the loading path values are listed in Table 3.

Modify $p_{\max }^{*}$ to $188 \mathrm{kPa}$ according to the load path, and when $\alpha=1$, the calculation results of the fuzzy plastic Cambridge model are presented in Table 4.

The plastic strain results calculated using the fuzzy plastic Cambridge model for varied parameters of the plastic membership function are depicted in Figure 8.

As shown in Figure 8, the plastic strain of the soil material can be easily calculated according to the fuzzy plastic Cambridge model in the original elastic region. When the shear strain and $\mathrm{q} / \mathrm{p}$ are stable, the larger the plastic membership function parameter, the smaller the plastic body strain. However, when In $p$ is stable, the larger the plastic membership function parameter, the larger the plastic body strain.

Figure 9 depicts that the plastic body strain and plastic shear strain change continuously with a continuous change in plastic membership function; moreover, the size is controlled by the plastic membership function's value. The greater the value of the plastic membership function, the greater the plastic shear and plastic body strains. Notably, the plastic membership function can effectively reflect the plastic strain of the stress point in the original elastic region, and there is a one-to-one correspondence between the plastic strain and the degree of plastic membership.

3.3. Cyclic Loading and Unloading. Using the soil sample listed in Table 2, the axial cyclic loading and unloading were increased for the test under certain confining pressure conditions. To exhibit the plastic strain inside the initial yield surface, cyclic loading and unloading action were applied between a fuzzy yield surface $(0<\mu<1)$ and the initial yield surface $(\mu=1)$. The axial dynamic load adopts the form of a sine wave with a frequency of $1 \mathrm{~Hz}$. The law of loading and unloading is defined by

$$
q(t)=q_{j}+q_{d} * \sin \left(2 \pi t+\frac{3}{2} \pi\right)
$$

where $q(t)$ represents the magnitude of the dynamic load at time $t$ and $q_{d}$ represents the dynamic load amplitude.

The initial consolidation pressure of the test soil was $200 \mathrm{kPa}$, and the values of the cyclic loading are listed in Table 5.

The stress-strain curves calculated using the fuzzy plastic model are shown in Figure 10.

Figure 10 indicates that different initial confining pressures correspond to different plastic membership function values, affecting the cyclic loading and unloading results. When the initial confining pressure is stable, the cyclic loading and unloading curves increase for an increase in the plastic membership function value. Moreover, when the plastic membership function value is stable, the increase in the initial confining pressure increases the cyclic loading and unloading curves. The cyclic loading and unloading path is clear, and its mathematical expression is simple; therefore, fuzzy plasticity theory can solve the problem of cyclic loading and unloading. The loading and unloading path can be noticed using the fuzzy cone surface; thus, the fuzzy plastic theory has more advantages than the classical plastic theory in the cyclic loading and unloading problem. 

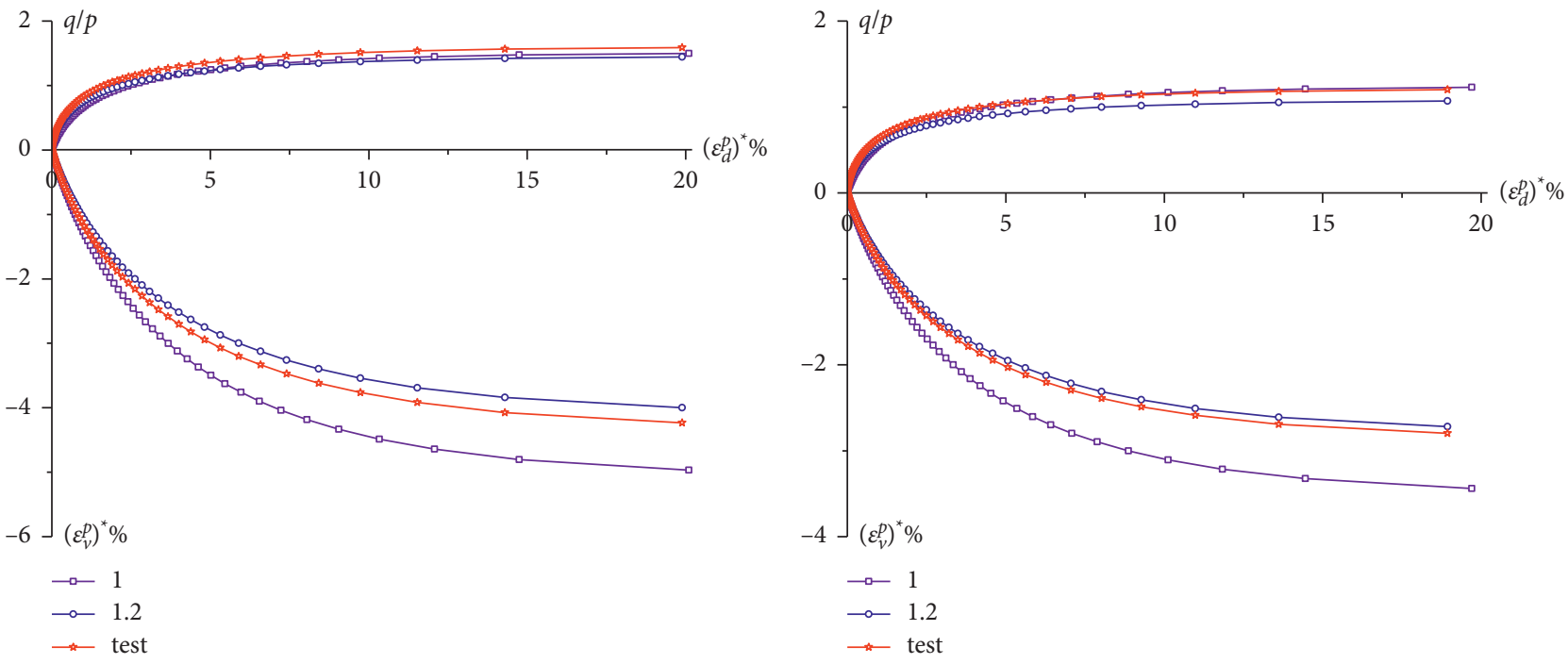

(a)

(b)

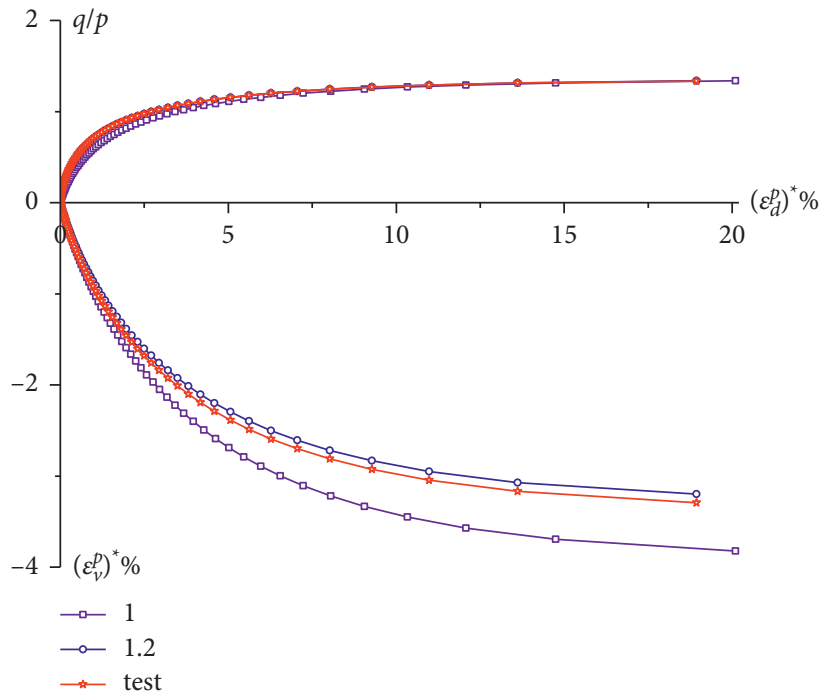

(c)

FIgURE 7: Test comparison curve of $q / p-\left(\varepsilon_{v}^{p}\right)^{*}-\left(\varepsilon_{d}^{p}\right)^{*}$ for different confining pressures. (a) $30 \mathrm{kpa}$. (b) $60 \mathrm{kpa}$. (c) $90 \mathrm{kpa}$.

TABLE 3: Load path table.

\begin{tabular}{lccc}
\hline$p$ & $q$ & $\eta=q / p$ & $p_{\max }^{*}$ \\
\hline 1.5942 & 0.001594191 & 0.001 & 1.595390284 \\
2.7742 & 0.065563384 & 0.024 & 2.823927555 \\
3.9542 & 0.182947243 & 0.046 & 4.094166046 \\
5.1342 & 0.353745768 & 0.069 & 5.407175311 \\
6.3142 & 0.577958959 & 0.092 & 6.764049334 \\
7.4942 & 0.855586815 & 0.114 & 8.165907053 \\
8.6742 & 1.186629338 & 0.137 & 9.613892893 \\
9.8542 & 1.571086526 & 0.159 & 11.10917731 \\
11.0342 & 2.00895838 & 0.182 & 12.65295736 \\
12.2142 & 2.500244901 & 0.205 & 14.24645723 \\
13.3942 & 3.044946087 & 0.227 & 15.89092888 \\
14.5742 & 3.643061938 & 0.250 & 17.58765257 \\
15.7542 & 4.294592456 & 0.273 & 19.33793751 \\
16.9342 & 4.99953764 & 0.295 & 21.14312244 \\
\hline
\end{tabular}


TABle 3: Continued.

\begin{tabular}{|c|c|c|c|}
\hline$p$ & $q$ & $\eta=q / p$ & $p_{\max }^{*}$ \\
\hline 18.1142 & 5.757897489 & 0.318 & 23.00457629 \\
\hline 19.2942 & 6.569672005 & 0.340 & 24.9236988 \\
\hline 20.4742 & 7.434861186 & 0.363 & 26.9019212 \\
\hline 21.6542 & 8.353465033 & 0.386 & 28.94070684 \\
\hline 22.8342 & 9.325483546 & 0.408 & 31.04155191 \\
\hline 24.0142 & 10.35091673 & 0.431 & 33.20598609 \\
\hline 25.1942 & 11.42976457 & 0.454 & 35.43557332 \\
\hline 26.3742 & 12.56202708 & 0.476 & 37.73191248 \\
\hline 27.5542 & 13.74770426 & 0.499 & 40.09663813 \\
\hline 28.7342 & 14.9867961 & 0.522 & 42.53142129 \\
\hline 29.9142 & 16.27930261 & 0.544 & 45.03797019 \\
\hline 31.0942 & 17.62522378 & 0.567 & 47.61803106 \\
\hline 32.2742 & 19.02455962 & 0.589 & 50.27338894 \\
\hline 33.4542 & 20.47731013 & 0.612 & 53.00586846 \\
\hline 34.6342 & 21.9834753 & 0.635 & 55.81733475 \\
\hline 35.8142 & 23.54305514 & 0.657 & 58.70969419 \\
\hline 36.9942 & 25.15604964 & 0.680 & 61.68489538 \\
\hline 38.1742 & 26.82245881 & 0.703 & 64.74492995 \\
\hline 39.3542 & 28.54228265 & 0.725 & 67.89183351 \\
\hline 40.5342 & 30.31552115 & 0.748 & 71.12768653 \\
\hline 41.7142 & 32.14217431 & 0.771 & 74.45461534 \\
\hline 42.8942 & 34.02224215 & 0.793 & 77.87479303 \\
\hline 44.0742 & 35.95572465 & 0.816 & 81.39044046 \\
\hline 45.2542 & 37.94262181 & 0.838 & 85.00382727 \\
\hline 46.4342 & 39.98293364 & 0.861 & 88.71727286 \\
\hline 47.6142 & 42.07666014 & 0.884 & 92.53314749 \\
\hline 48.7942 & 44.2238013 & 0.906 & 96.45387327 \\
\hline 49.9742 & 46.42435713 & 0.929 & 100.4819253 \\
\hline 51.1542 & 48.67832762 & 0.952 & 104.6198328 \\
\hline 52.3342 & 50.98571278 & 0.974 & 108.87018 \\
\hline 53.5142 & 53.34651261 & 0.997 & 113.2356078 \\
\hline 54.6942 & 55.7607271 & 1.019 & 117.7188144 \\
\hline 55.8742 & 58.22835626 & 1.042 & 122.3225567 \\
\hline 57.0542 & 60.74940008 & 1.065 & 127.0496517 \\
\hline 58.2342 & 63.32385857 & 1.087 & 131.9029774 \\
\hline 59.4142 & 65.95173173 & 1.110 & 136.8854745 \\
\hline 60.5942 & 68.63301955 & 1.133 & 142.0001471 \\
\hline 61.7742 & 71.36772204 & 1.155 & 147.2500648 \\
\hline 62.9542 & 74.15583919 & 1.178 & 152.6383634 \\
\hline 64.1342 & 76.99737101 & 1.201 & 158.1682463 \\
\hline 65.3142 & 79.89231749 & 1.223 & 163.8429866 \\
\hline 66.4942 & 82.84067864 & 1.246 & 169.6659275 \\
\hline 67.6742 & 85.84245446 & 1.268 & 175.6404848 \\
\hline 68.5656 & 88.52504486 & 1.291 & 181.0082873 \\
\hline 69.0256 & 89.905844 & 1.303 & 183.7912786 \\
\hline 69.1500 & 92.169 & 1.330 & 188 \\
\hline
\end{tabular}

TABLE 4: Calculation results when $\alpha=1$.

\begin{tabular}{lcccc}
\hline$\eta=q / p$ & $p_{\max }^{*}$ & $\mu$ & $\left(\varepsilon_{v}^{p}\right)^{*}$ & $\left(\varepsilon_{d}^{p}\right)^{*}$ \\
\hline 0.001 & 1.595390284 & 0.008486119 & 0 & 0 \\
0.024 & 2.823927555 & 0.015020891 & -0.000269363 & 0.000206193 \\
0.046 & 4.094166046 & 0.021777479 & -0.000547907 & 0.000423172 \\
0.069 & 5.407175311 & 0.028761571 & -0.000835868 & 0.000651513 \\
0.092 & 6.764049334 & 0.035978986 & -0.001133486 & 0.000891825 \\
0.114 & 8.165907053 & 0.043435676 & -0.001441009 & 0.001144757 \\
0.137 & 9.613892893 & 0.051137728 & -0.00175869 & 0.001410999 \\
0.159 & 11.10917731 & 0.059091369 & -0.002086785 & 0.001691287 \\
0.182 & 12.65295736 & 0.067302965 & -0.002425559 & 0.001986403 \\
\hline
\end{tabular}


TABle 4: Continued.

\begin{tabular}{|c|c|c|c|c|}
\hline$\eta=q / p$ & $p_{\max }^{*}$ & $\mu$ & $\left(\varepsilon_{v}^{p}\right)^{*}$ & $\left(\varepsilon_{d}^{p}\right)^{*}$ \\
\hline 0.205 & 14.24645723 & 0.075779028 & -0.002775281 & 0.002297185 \\
\hline 0.227 & 15.89092888 & 0.084526217 & -0.003136229 & 0.002624525 \\
\hline 0.250 & 17.58765257 & 0.093551343 & -0.003508684 & 0.00296938 \\
\hline 0.273 & 19.33793751 & 0.10286137 & -0.003892934 & 0.003332772 \\
\hline 0.295 & 21.14312244 & 0.112463417 & -0.004289276 & 0.003715797 \\
\hline 0.318 & 23.00457629 & 0.122364767 & -0.00469801 & 0.004119632 \\
\hline 0.340 & 24.9236988 & 0.132572866 & -0.005119446 & 0.00454554 \\
\hline 0.363 & 26.9019212 & 0.143095326 & -0.005553899 & 0.004994881 \\
\hline 0.386 & 28.94070684 & 0.15393993 & -0.006001692 & 0.005469121 \\
\hline 0.408 & 31.04155191 & 0.165114638 & -0.006463155 & 0.005969839 \\
\hline 0.431 & 33.20598609 & 0.176627586 & -0.006938624 & 0.006498746 \\
\hline 0.454 & 35.43557332 & 0.188487092 & -0.007428446 & 0.00705769 \\
\hline 0.476 & 37.73191248 & 0.200701662 & -0.007932972 & 0.007648678 \\
\hline 0.499 & 40.09663813 & 0.21327999 & -0.008452562 & 0.008273888 \\
\hline 0.522 & 42.53142129 & 0.226230964 & -0.008987587 & 0.008935692 \\
\hline 0.544 & 45.03797019 & 0.239563671 & -0.009538421 & 0.009636677 \\
\hline 0.567 & 47.61803106 & 0.253287399 & -0.010105451 & 0.010379673 \\
\hline 0.589 & 50.27338894 & 0.267411643 & -0.01068907 & 0.011167779 \\
\hline 0.612 & 53.00586846 & 0.281946109 & -0.01128968 & 0.0120044 \\
\hline 0.635 & 55.81733475 & 0.296900717 & -0.011907693 & 0.012893286 \\
\hline 0.657 & 58.70969419 & 0.312285607 & -0.012543529 & 0.01383858 \\
\hline 0.680 & 61.68489538 & 0.328111146 & -0.013197618 & 0.01484487 \\
\hline 0.703 & 64.74492995 & 0.344387925 & -0.013870399 & 0.015917259 \\
\hline 0.725 & 67.89183351 & 0.361126774 & -0.014562321 & 0.017061436 \\
\hline 0.748 & 71.12768653 & 0.378338758 & -0.015273843 & 0.018283772 \\
\hline 0.771 & 74.45461534 & 0.396035188 & -0.016005434 & 0.01959143 \\
\hline 0.793 & 77.87479303 & 0.414227622 & -0.016757573 & 0.020992496 \\
\hline 0.816 & 81.39044046 & 0.432927875 & -0.01753075 & 0.022496147 \\
\hline 0.838 & 85.00382727 & 0.452148017 & -0.018325466 & 0.024112847 \\
\hline 0.861 & 88.71727286 & 0.471900388 & -0.019142232 & 0.0258546 \\
\hline 0.884 & 92.53314749 & 0.492197593 & -0.019981571 & 0.027735261 \\
\hline 0.906 & 96.45387327 & 0.513052517 & -0.020844017 & 0.029770933 \\
\hline 0.929 & 100.4819253 & 0.534478326 & -0.021730117 & 0.031980474 \\
\hline 0.952 & 104.6198328 & 0.556488472 & -0.022640428 & 0.034386158 \\
\hline 0.974 & 108.87018 & 0.579096702 & -0.023575519 & 0.037014543 \\
\hline 0.997 & 113.2356078 & 0.602317063 & -0.024535975 & 0.039897639 \\
\hline 1.019 & 117.7188144 & 0.626163906 & -0.025522389 & 0.043074497 \\
\hline 1.042 & 122.3225567 & 0.650651897 & -0.026535371 & 0.046593422 \\
\hline 1.065 & 127.0496517 & 0.67579602 & -0.02757554 & 0.050515137 \\
\hline 1.087 & 131.9029774 & 0.701611582 & -0.028643533 & 0.054917416 \\
\hline 1.110 & 136.8854745 & 0.728114226 & -0.029739998 & 0.059902102 \\
\hline 1.133 & 142.0001471 & 0.755319932 & -0.030865597 & 0.065606152 \\
\hline 1.155 & 147.2500648 & 0.783245026 & -0.032021009 & 0.072219839 \\
\hline 1.178 & 152.6383634 & 0.811906188 & -0.033206923 & 0.080018488 \\
\hline 1.201 & 158.1682463 & 0.841320459 & -0.034424048 & 0.089421975 \\
\hline 1.223 & 163.8429866 & 0.871505248 & -0.035673105 & 0.101117263 \\
\hline 1.246 & 169.6659275 & 0.902478338 & -0.036954832 & 0.116345697 \\
\hline 1.268 & 175.6404848 & 0.934257898 & -0.038269982 & 0.13771866 \\
\hline 1.291 & 181.0082873 & 0.962810039 & -0.039452315 & 0.168112812 \\
\hline 1.303 & 183.7912786 & 0.977613184 & -0.04006385 & 0.190350448 \\
\hline 1.330 & 188 & 1 & -0.04124346 & 0.192589561 \\
\hline
\end{tabular}




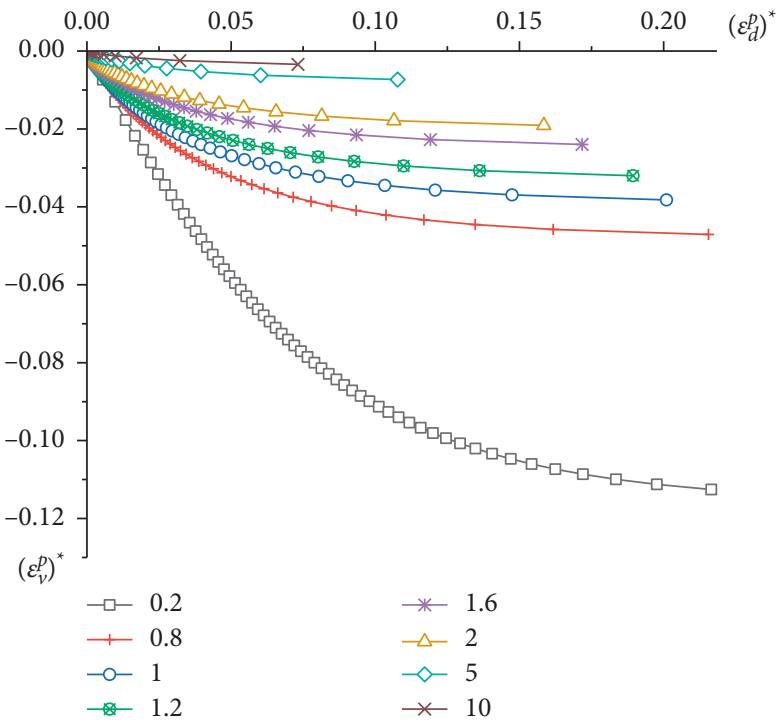

(a)

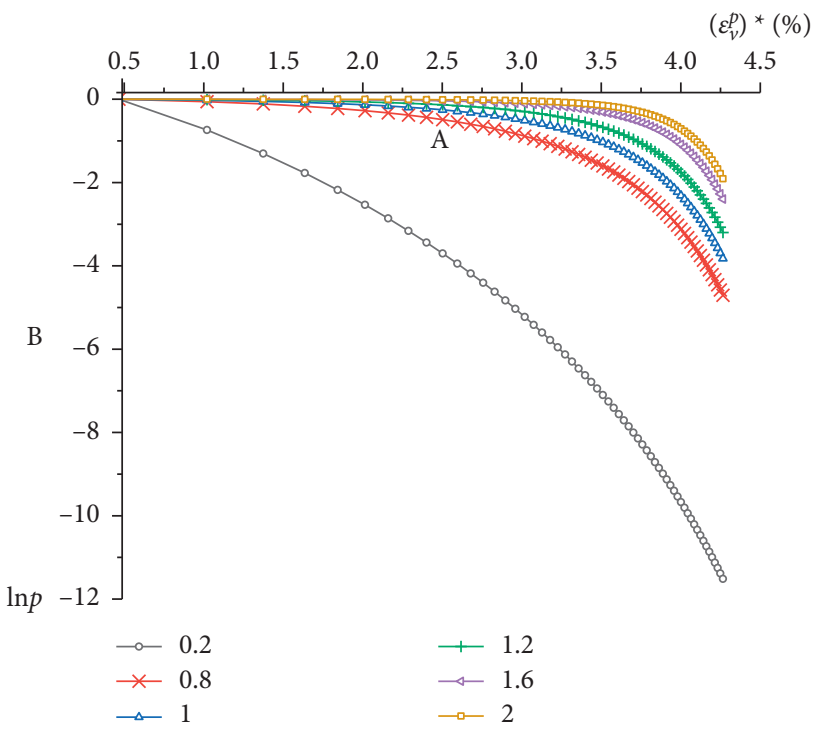

(b)

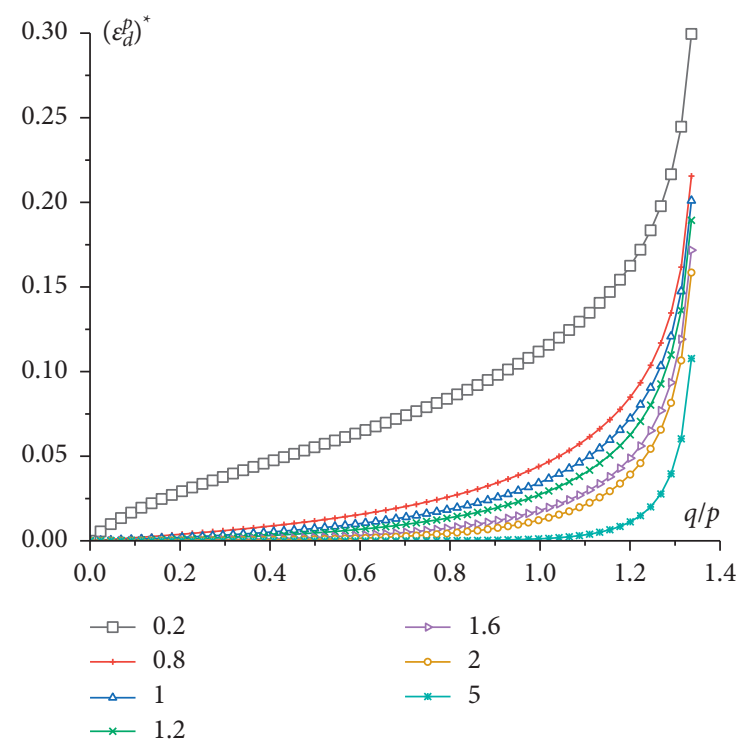

(c)

Figure 8: The calculation result curve of the fuzzy Cambridge model. (a) Curve of $\left(\varepsilon_{d}^{p}\right) *-\left(\varepsilon_{v}^{p}\right) *$. (b) Curve of $\ln p-\left(\varepsilon_{v}^{p}\right)^{*}$. (c) Curve of $q / p-\left(\varepsilon_{d}^{p}\right)^{*}$.

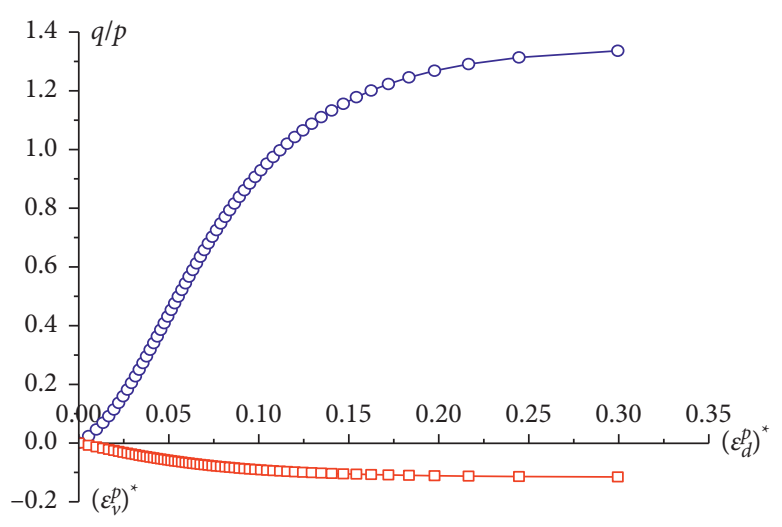

(a)

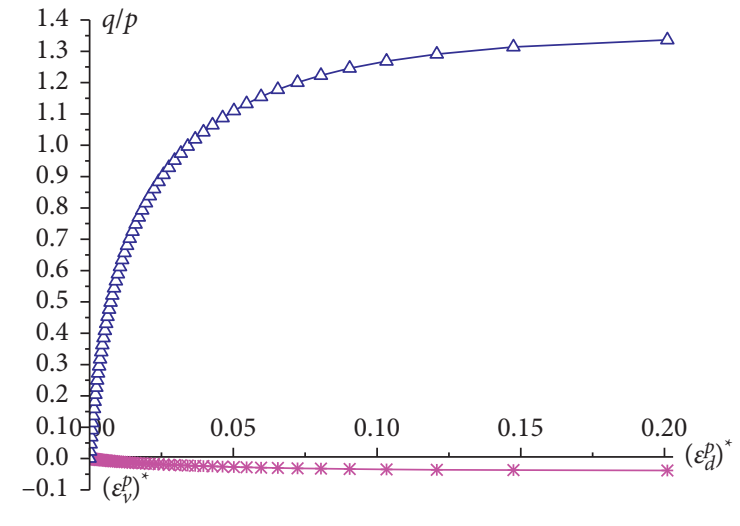

(b)

FIgure 9: Continued. 


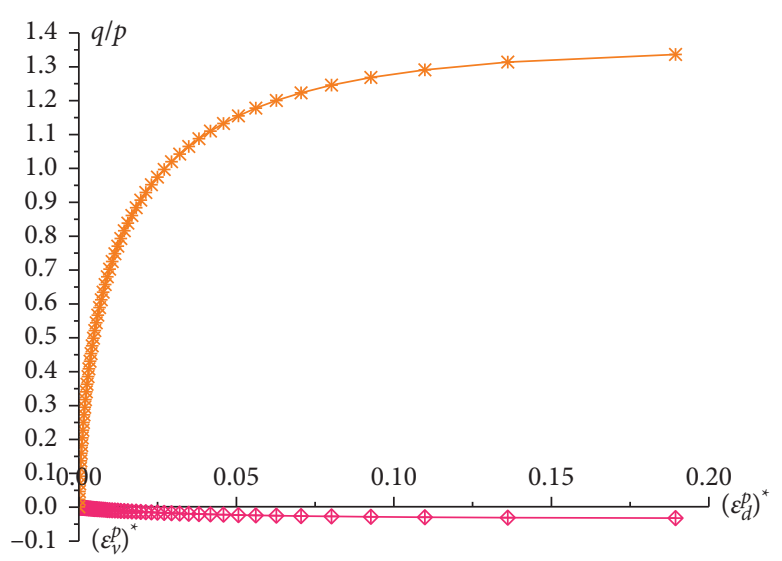

(c)

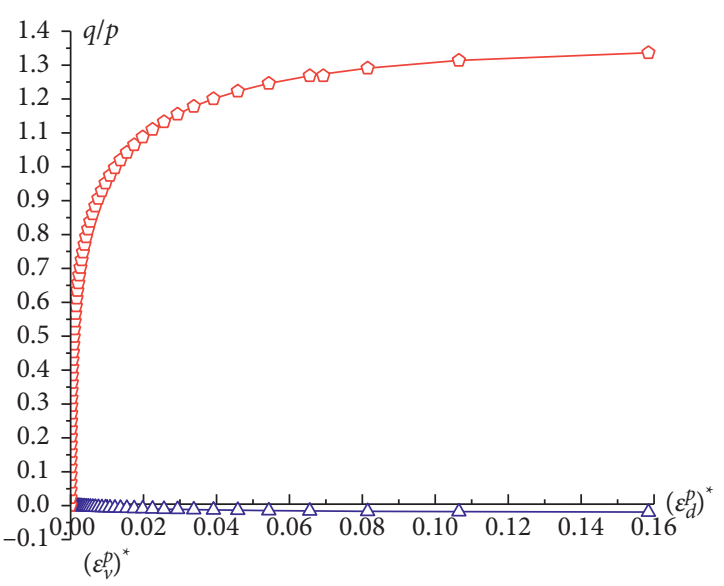

(d)

Figure 9: Relationship between plastic membership function and stress-strain. (a) $\alpha=0.2$. (b) $\alpha=1$. (c) $\alpha=1.2$. (d) $\alpha=2$.

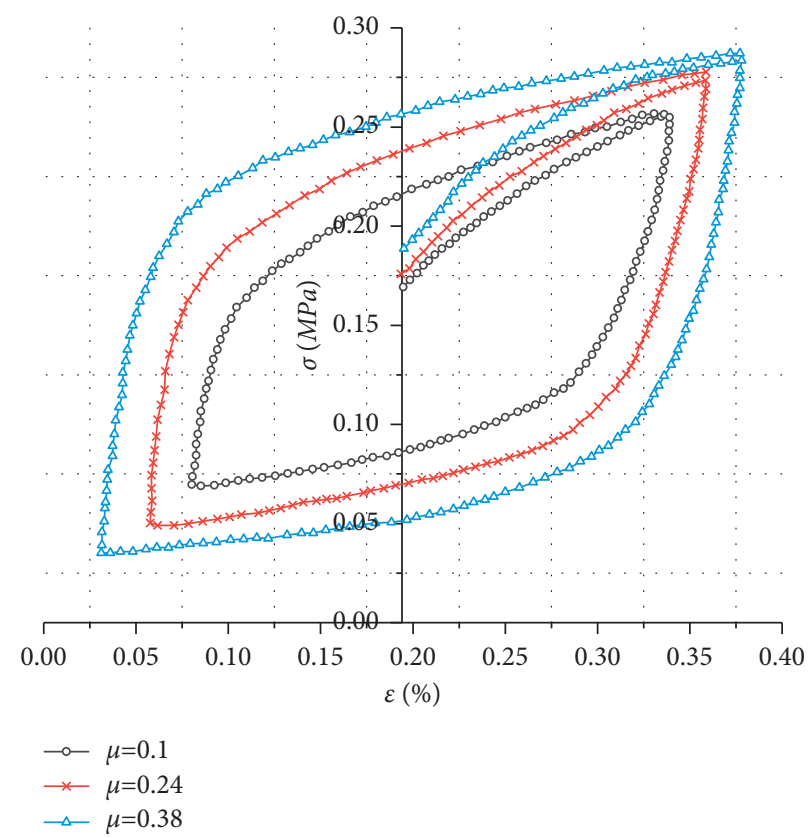

FIGURE 10: Cycle loading and unloading curves.

TABLE 5: Cyclic load amplitude values.

\begin{tabular}{lcccc}
\hline$p$ & $q_{j}$ & $q_{d}$ & $\mu$ & $\alpha$ \\
\hline 90 & 145 & 55 & 0.38 & 1.2 \\
60 & 130 & 70 & 0.24 & 1.2 \\
30 & 115 & 85 & 0.10 & 1.2 \\
\hline
\end{tabular}

\section{Discussion}

The core of the fuzzy plasticity theory is to select an appropriate plastic membership function. This study adopts the assignment-type plastic membership function form; however, it cannot accurately reflect the plasticity of the material. The subsequent step of the study uses an inversion analysis to determine a more appropriate membership function.

\section{Conclusion}

This study combined fuzzy mathematics with the Cambridge model and used the associated flow law to obtain a fuzzy plastic Cambridge model. The model reflected the plastic strain of the stress point in the classical initial yield plane and the unobvious characteristics of the soil's yield point. The fuzzy plastic Cambridge model controlled the plastic strain 
according to the degree of plastic membership. By comparing and analyzing the fuzzy Cambridge model and the triaxial test results, the model parameters were determined, and the reliability of the fuzzy plastic Cambridge model was verified. The fuzzy plasticity theory considered elastoplasticity. In the cyclic loading and unloading process, the change in the fuzzy cone surface indicated the loading and unloading paths. The mathematical expressions in the loading and unloading processes were simple, and the loading path was clear. This proved that the fuzzy plastic model is appropriate for solving the cyclic loading and unloading problems. The fuzzy plasticity theory is based on fuzzy mathematics and classical plasticity models. Most plastic models can be transformed into fuzzy plastic constitutive models because there is no need to build a new plastic constitutive model. Therefore, fuzzy plasticity theory is easy to popularize.

\section{Data Availability}

The data used to support the findings of this study are included within the article.

\section{Conflicts of Interest}

The authors declare that they have no conflicts of interest.

\section{Acknowledgments}

This research was funded by Liaoning Province Doctor Startup Fund Program by Department of Science and Technology of Liaoning Province (Grant no. 20180540101).

\section{References}

[1] K. Hashiguchi, Elastoplastic Constitutive Laws of Granular Material. Constitutive Equations of Soils, JSSMFE, Tokyo, Japan, 1977.

[2] Y. F. Dafalias, "Bounding surface plasticity. I: mathematical foundation and hypoplasticity," Journal of Engineering $\mathrm{Me}$ chanics, vol. 112, no. 9, pp. 966-987, 1986.

[3] Y. F. Dafalias and L. R. Herrmann, "Bounding surface plasticity. II: application to isotropic cohesive soils," Journal of Engineering Mechanics, vol. 112, no. 12, pp. 1263-1291, 1986.

[4] A. Anandarajah and Y. F. Dafalias, "Bounding surface plasticity. III: application to anisotropic cohesive soils," Journal of Engineering Mechanics, vol. 112, no. 12, pp. 1292-1318, 1986.

[5] M. Klisinski, "Plasticity theory based on fuzzy sets," Journal of Engineering Mechanics, vol. 114, no. 4, pp. 563-582, 1988.

[6] S. Jiang and S. Xue, "Analysis of fuzzy factors in elastoplastic mechanics," Mechanics in Engineering, vol. 46, no. 4, pp. 37-38, 1989.

[7] M. Fu, B. Xu, and Z. Xiong, "Problem of fuzzy elasto-viscoplasticity and uniqueness and existence of the solution," Journal of Jiangsu Polytechnic University, vol. 14, no. 1, pp. 1-11, 1992.

[8] M. Fu, Z. Xiong, and B. Xu, "Analysis of fuzzy elasto-viscoplasticity for spherical shell," Engineering Mechanics, vol. 11, no. 2, pp. 1-7, 1994.

[9] X. Wang and M. Fu, "Fuzzy elasto-visco-plasticity analysis of finite deformation based on the L-D plastic flow rule," Applied Mathematics and Mechanics, vol. 36, no. 2, pp. 128-139, 2015.
[10] X. Wang, M. Fu, and X. Hu, "Bounding surface model based on membership function under cyclic loading," Journal of Harbin Engineering University, vol. 36, no. 12, pp. 1560-1564, 2015.

[11] X. Wang and M. Fu, "Research for constitutive model of fuzzy elastic-plasticity by membership function," Journal of $\mathrm{Vi}$ bration and Shock, vol. 34, no. 23, pp. 115-120, 2015.

[12] X. Shi, Z. Cheng, and L. Wang, "Dentification of geo-material constitutive model using fuzzy system," Hydro-Science and Engineering, vol. 33, no. 3, pp. 10-16, 2010.

[13] D. Liu and H. Li, "Application of applied mathematics and fuzzy mathematics in geotechnical engineering," Chinese Journal of Geotechnical Engineering, vol. 41, no. 10, p. 1977, 2019.

[14] Z. Fu and T. Liu, "Lope stability analysis based on the renormalization group and fuzzy probability method," Journal of Transport Science and Engineering, vol. 31, no. 2, pp. 29-33, 2015.

[15] L. Zhang, E. Chi, M. Zhao, T. Tao, and Y. Li, “Application of fuzzy comprehensive evaluation in rock-soil blasting safety assessment," Engineering Blasting, vol. 21, no. 2, pp. 13-17, 2015.

[16] J. Li, S. Chen, and L. Jiang, "An improved bounding surface model for clay under cyclic loading," Rock and Soil Mechanics, vol. 36, no. 02, pp. 387-392+450, 2015.

[17] C. Zhao, G. Cai, C. Zhao, and W. Zhan, "Cyclic constitutive model of saturated sand based on the bounding surface theory," Chinese Journal of Solid Mechanics, vol. 38, no. 3, pp. 244-252, 2017.

[18] Y. Zhou, Q. Sheng, X. Leng, Z. Zhu, and X. Fu, "Preliminary application of subloading surface to cyclic plastic model for rock under cyclic loading," Chinese Journal of Rock Mechanics and Engineering, vol. 34, no. 10, pp. 2073-2082, 2015.

[19] M. Huang, C. Yang, and Y. Cui, "Elasto-plastic bounding surface model for unsaturated soils under cyclic loading," Chinese Journal of Geotechnical Engineering, vol. 31, no. 6, pp. 817-823, 2009.

[20] H. Yao and J. Wang, "An improved kinematic hardening bounding surface model for saturated clay," Journal of Tianjin University, vol. 50, no. 12, pp. 1329-1336, 2017.

[21] X. Dong, C. Zhao, W. Zhang, and C. Zhao, "Cyclic loading boundary surface model of saturated sand based on phase transition state," Industrial Construction, vol. 46, no. 07, pp. 129-133+139, 2016. 